\title{
Interrelación entre el contenido de metabolitos secundarios de las especies Gliricidia sepium y Tithonia diversifolia y algunas propiedades físicoquímicas del suelo
}

\author{
Interrelation between the content of secondary \\ metabolites of species Gliricidia sepium and Tithonia \\ diversifolia and some physicochemical properties of soil
}

\author{
'Luz Elena Santacoloma Varón y 2Jairo Enrique Granados \\ 'Zootecnista; especialista en nutrición animal sostenible; magíster en gestión ambiental para el desarrollo sostenible. \\ ${ }^{2}$ Licenciado en química; magíster en ciencias químicas; magíster en docencia de la química. \\ 1, 2Universidad Nacional Abierta y a Distancia (UNAD); Escuela de Ciencias Agrícolas, Pecuarias y del Medio Ambiente \\ (ECAPMA). Bogotá, Colombia. \\ 1'luz.santacoloma@unad.edu.co, 2jairo.granados@unad.edu.co
}

\begin{abstract}
Resumen
Se recolectaron muestras de dos plantas forrajeras (hoja peciolo) de las especies Gliricidia sepium y Tithonia diversifolia, en diferentes condiciones edáficas (departamentos del Valle del Cauca y Cesar), y se evaluó su contenido de polifenoles totales, taninos totales, taninos condensados y saponinas. El propósito fue detectar el efecto de las condiciones de conductividad eléctrica sobre la concentración de estas fitobiomoléculas secundarias metabolizadas por la célula vegetal. Se aplicaron técnicas analíticas e instrumentales de la AOAC (2005) para determinar la presencia de dichos fitometabolitos secundarios. Los resultados obtenidos para cada variable se sometieron a un análisis de varianza en doble vía, con el fin de determinar diferencias estadísticas entre zonas muestreadas y especies evaluadas. Adicionalmente, se realizó un análisis de correlación múltiple, utilizando el coeficiente de Pearson, con el propósito de detectar las posibles correlaciones entre conductividad eléctrica, y el contenido de los metabolitos secundarios cuantificados en las especies muestreadas. Se concluyó que el contenido de polifenoles totales, taninos totales, taninos condensados y saponinas no presentó diferencias estadísticamente significativas entre las tres zonas muestreadas, y se presentó una alta correlación entre el valor de la conductividad eléctrica del suelo y el contenido de metabolitos secundarios.
\end{abstract}

Palabras clave: Metabolitos secundarios, polifenoles, taninos totales, taninos condensados, saponinas, conductividad eléctrica.

\begin{abstract}
Samples were collected from 2 forage plants (leaf stalk) of the species Gliricidia sepium and Tithonia diversifolia in different soil conditions (departments of Valle del Cauca and Cesar) and evaluated for total polyphenol content, total tannins, condensed tannins and Saponinas. The purpose was to detect the effect of the conditions of electrical conductivity on the concentration of these secondary fitobiomoléculas metabolized by the plant cell. We applied analytical techniques and instrumental AOAC (2005) to determine the presence of such fitometabolitos side. The results obtained for each variable were subjected to analysis of variance two-way, in order to determine statistical differences between areas sampled and evaluated species. Additionally, we performed a multiple correlation analysis using the Pearson coefficient, in order to detect any correlation between electrical conductivity and the content of secondary metabolites quantified in the species sampled. It was concluded that the content of total polyphenols, total tannins, condensed tannins and saponins did not show statistically significant differences between the three areas sampled, and there was high correlation between the value of the electrical conductivity of soil and content of secondary metabolites.
\end{abstract}

Key words: Secondary metabolites, polyphenols, tannins overall, condensed tannins, saponins, electrical conductivity. 


\section{Introducción}

El uso de leguminosas y de asteráceas en sistemas de alimentación animal es una práctica que se ha venido implementando desde hace varios años en muchas regiones del trópico, por el alto contenido de proteína y de minerales que contienen. Entre las leguminosas se destaca la arbórea Gliricidia sepium, especie poco valorada como fuente suplementaria de proteína; en especial, durante la época seca, cuando los forrajes son escasos y de mala calidad (Palma et al., 1999). Entre las asteráceas sobresale la Tithonia diversifolia, planta de gran adaptabilidad a diversos pisos térmicos y con alto contenido de nutrientes para rumiantes y no rumiantes.

No obstante lo anterior, es importante tener en cuenta un factor que, en cierta medida, puede constituir una limitante en el uso de estas plantas como material forrajero, y es la presencia de metabolitos secundarios que pueden afectar la digestibilidad, y los cuales no solo pueden interferir con los procesos de digestión y absorción de los nutrientes, sino que, previamente a su paso al torrente sanguíneo, pueden desencadenar variados efectos sistémicos en los animales (Jackson et al., 1996).

El contenido de varios tipos de dichos metabolitos secundarios está influenciado por el genotipo de la planta (la especie y la variedad), las características ambientales (la radiación solar y la disponibilidad de agua), la velocidad de crecimiento, la madurez, la condición nutricional del suelo, la depredación y las enfermedades (Waterman y Mole, 1994). Así mismo, la aparición de los compuestos secundarios está relacionada con los mecanismos de defensa de la planta y los efectos del suelo y del clima (Harborne, 1993).

Según plantea Kumar (1997), los distintos compuestos que puede producir una especie presentan una determinada distribución dentro de los órganos, los tejidos y las células de una planta, y ello responde a menudo a las influencias ambientales. Según Norton (1997), los factores ambientales que mayor incidencia tienen en el contenido de taninos son la estación climática, la humedad ambiental y la luminosidad.

Entre los metabolitos secundarios se destacan los taninos, por su abundancia en la naturaleza, y, particularmente, en los forrajes; Posada (2005) los define como un grupo de polifenoles solubles en agua, de relativo alto peso molecular y con presencia en casi todas las plantas; particularmente, en las dicotiledóneas, de las cuales hacen parte las leguminosas. Leinmúller (1995) explica que la distribución de metabolitos secundarios al interior de las plantas varía entre especies, y dentro de la célula vegetal se localizan en las vacuolas citoplasmáticas o en la pared celular.

La síntesis de taninos, por su parte, se presenta a partir de esqueletos de carbono del metabolismo primario, como ocurre con los rebrotes más jóvenes de la planta, que también necesitan las reservas de carbono para su crecimiento (Haukioja, Niemela y Sirén, 1995).
Así mismo, los taninos tienen la capacidad para formar enlaces químicos más o menos estables al interactuar con proteínas, carbohidratos y otras macromoléculas de los alimentos, o con las enzimas digestivas; además, se ha demostrado que los taninos pueden ser tóxicos para las bacterias; en los rumiantes este efecto se refleja en cambios de la digestión ruminal de proteínas y carbohidratos, y en la producción de ácidos grasos volátiles (Makkar et al., 1995).

Otro aspecto inherente a la planta y que incide en el contenido de taninos, es la parte de la planta a la cual pertenece, por lo cual, se ha determinado que el contenido de dichos metabolitos en el peciolo es diferente del que hay en la hoja. Por su parte, Oncina et al. (2000) han demostrado que las partes de la planta presentan distintas productividades de metabolitos secundarios, y que se acumulan cantidades de metabolitos diferentes de acuerdo con la parte de la planta a la cual corresponde tal acumulación.

Los taninos pueden dividirse, según su estructura química, en taninos condensados (proantocianidinas) y taninos hidrolizables (derivados de los ácidos gálico y elágico). Al respecto, Romero y Palma (2001) encontraron que factores como el pastoreo provocan una disminución en el contenido de taninos condensados y de los fenoles totales en Gliricidia sepium; probablemente, porque se presenta una mayor competición por reservas para la generación de hojas, más que para la producción de taninos condensados.

El efecto de la época también varió según la naturaleza del tanino, sin mostrar tendencias consistentes. Los mismos autores reportan que los taninos adheridos a proteína representaron alrededor del $70 \%-75 \%$ de los taninos condensados totales; los valores son más altos durante la época de sequía que en la de lluvias. Los taninos condensados pueden llegar a producir efectos depresivos sobre el consumo y la digestibilidad de la materia seca y el nitrógeno, pues provocan saciedad y limitan, por lo tanto, el consumo de materia seca (Flores et al., 1999; Gutiérrez et al., 2003).

Gershenzon (1984) reporta que la disponibilidad de agua contribuye a la producción de taninos condensados; al encontrarse en periodo de escasez de agua, las plantas cierran sus estomas y restringen el proceso de fotosíntesis. De este modo se esperaría una relación negativa entre el estrés hídrico y la producción de compuestos fenólicos.

Por su parte, la leguminosa G. sepium puede ser considerada como una de las leguminosas tropicales con menor contenido de taninos condensados, en comparación con otras como Flemingia macrophylla, con (388 g/kg) (Cano, Carulla y Lascano, 1994), Desmodium ovalifolium (238 g/ $\mathrm{kg})$, Acacia mangium (100 g/kg), Callyandra sp (194 g/kg) (Jackson y Barry, 1996). Esta concentración bien podría explicar el comportamiento de los animales de desarrollar un grado de gustosidad medio para G. sepium (Kaito et al., 1996).

Por lo anterior, y considerando todos los análisis químicos realizados, la hoja de Gliricidia sepium es un excelente forraje para dietas tropicales, pues presenta los compuestos 
nutricionales más altos, una buena tasa de degradabilidad y principios tóxicos más bajos que los de otros forrajes estudiados.

Respecto a la especie forrajera Tithonia diversifolia, en un análisis de metabolitos secundarios realizado por Rosales (1998) no se encontraron ni fenoles ni taninos, mientras que Vargas (1994) halló un bajo contenido de fenoles y ausencia de saponinas. Mungarulire et al. (1993) encontraron en esta especie un compuesto citotóxico. También se cree que Tithonia diversifolia destruye los parásitos intestinales del ganado, y que sus residuos amargos ayudan a la digestión del material fibroso (Wanjau et al., 1998).

Las saponinas son otro tipo de metabolitos secundarios, conformados por compuestos que en forma glucosídica (como esteroides reguladores del crecimiento) pueden generar en las plantas alto crecimiento, desarrollo, rápida recuperación ante la poda y brotes abundantes (Ashok, Vincent y Nessler, 2000). También Kumar (1997) plantea que ante la dependencia de las concentraciones y de las estructuras químicas específicas, los compuestos saponínicos pueden constituir factores antinutricionales en rumiantes y monogástricos, por conferirles a los forrajes un sabor amargo; también, provocar espumas consistentes e interferir en la absorción de los alimentos. No obstante, también pueden tener un efecto positivo en el metabolismo digestivo, al generar complejos con otros metabolitos secundarios de características tóxicas (Makkar, 1997).

En consecuencia, es necesario realizar investigaciones sobre los efectos de los factores ambientales, tales como los edáficos, en la producción de metabolitos secundarios y en las interacciones suelo-planta, así como en la determinación de los factores que inciden en su concentración. Por lo tanto, el propósito central de la presente investigación fue establecer una interrelación específica entre algunas propiedades fisicoquímicas del suelo, como el $\mathrm{pH}$, y la conductividad eléctrica del suelo con la producción de metabolitos secundarios de las especies Gliricidia sepium y Tithonia sepium.

\section{Métodos}

\section{Zona de muestreo}

La recolección del material vegetal y de suelos se realizó en fincas ubicadas en el municipio de Tuluá (zona central del Valle del Cauca), y en los municipios de San Diego y de Pueblo Bello, en el Cesar, con las condiciones climáticas que se presentan en la Tabla 1.

Tabla 1. Condiciones climáticas de las regiones donde fueron tomadas las muestras de forraje

\begin{tabular}{lccc}
\hline Zonas de muestreo & \multicolumn{3}{c}{$\begin{array}{c}\text { Condiciones } \\
\text { climáticas }\end{array}$} \\
\hline & $\begin{array}{c}\text { Temperatura } \\
\text { promedio }\end{array}$ & $\begin{array}{c}\text { Precipitación } \\
\text { promedio } \\
\text { (mm lluvia) }\end{array}$ & $\begin{array}{c}\text { Altura } \\
\text { (msnm) }\end{array}$ \\
\hline Tuluá (Valle del & $\left({ }^{\circ} \mathbf{C}\right)$ & 1200 & 950 \\
Cauca) & 27 & 1311 & 500 \\
San Diego (Cesar) & 29 & - & 1200 \\
Pueblo Bello (Cesar) & 21 & & \\
\hline
\end{tabular}

Fuente: Plan de Desarrollo de los Municipios (2011).

\section{Recolección de las muestras}

La fracción vegetal (hojas y tallos tiernos) de Gliricidia sepium y de Tithonia diversifolia fue colectada a partir de plantas adultas en agosto de 2011, mes durante el cual predomina el tiempo seco en los tres lugares objeto de estudio. El material fue llevado al Laboratorio de Nutrición Animal de la sede Nacional José Celestino Mutis de la UNAD, y fue sometido a proceso de secado a temperatura ambiente, en un lugar ventilado, durante 72 horas. Posteriormente, las muestras fueron molidas y tamizadas, y se recolectaron sus extractos para el análisis de los metabolitos secundarios.

\section{Preparación y extracción del material para análisis fitoquímico}

Las muestras se secaron a la sombra durante 30 días; posteriormente se las molió hasta menos de $1 \mathrm{~mm}$ de tamaño de partícula, en un molino de martillo para laboratorio. Se prepararon 3 fracciones (etanol, éter dietílico y agua) con $10 \mathrm{~g}$ de cada muestra, $\mathrm{y}$, finalmente, se hizo un tamizaje fitoquímico). Por último, se cuantificaron las cantidades de polifenoles totales, taninos totales y taninos condensados presentes en las muestras, utilizando etanol como solvente y tomando como base las técnicas descritas por AOAC (1995). 


\section{Métodos analíticos para los fitometabolitos estudiados}

Los métodos utilizados se describen en la Tabla 2.

Tabla 2. Identificación de las variables analizadas y de la técnica analítica utilizada

\begin{tabular}{lll}
\hline \multicolumn{1}{c}{ Variable } & \multicolumn{1}{c}{ Técnica analítica } & \multicolumn{1}{c}{ Fuente } \\
\hline Polifenoles totales (PFT) & $\begin{array}{l}\text { Espectrofotometría con reactivo de } \\
\text { Folin Ciocalteu }\end{array}$ & Dewanto et al. (2002) \\
& $\begin{array}{ll}\text { Espectrofotometría con } \\
\text { Taninos condensados (TC) }\end{array}$ & Terrill et al. (1992) \\
Taninos totales & Folin-Denis y Folin Ciocalteu & Posada, et al. (2006) \\
\hline
\end{tabular}

Fuente: Laboratorio de Nutrición Animal UNAD (2011).

Para la prueba de las saponinas se tomó $1 \mathrm{~mL}$ de metanol y se le añadieron $9 \mathrm{~mL}$ de agua, para luego filtrarse dicha solución. Se tomó $1 \mathrm{~mL}$ del filtrado en un tubo de prueba pequeño, se lo agitó vigorosamente por 30 segundos y se lo dejó en reposo durante 15 minutos. La espuma sobrenadante indica la presencia de saponinas en el forraje. La proporción de saponinas se midió de acuerdo con la altura (h) de la espuma sobrenadante (Tabla 3).

Tabla 3. Determinación de saponinas según la altura de la espuma

\begin{tabular}{rrl}
\hline Rango $\mathbf{h}(\mathbf{m m})$ & \multicolumn{1}{c}{ Indicador } \\
\hline & 0,0 Prueba negativa \\
$0,1-5,0$ & Contenido muy bajo \\
$5,1-9,0$ & Contenido bajo \\
$9,1-14$ & Contenido moderado \\
Mayor que 14 & Contenido alto \\
\hline
\end{tabular}

Fuente: Cuéllar (1999).

Respecto a los análisis de $\mathrm{pH}$ y de conductividad eléctrica de las muestras de suelo, se utilizó la técnica descrita en IGAG (2006).

Se sometieron los resultados obtenidos para cada variable a un análisis de varianza en doble vía, con el fin de determinar diferencias estadísticas entre zonas muestreadas y especies evaluadas. Adicionalmente, se realizó un análisis de correlación múltiple, utilizando el coeficiente de Pearson, con el propósito de detectar las posibles correlaciones entre conductividad eléctrica, $\mathrm{pH}$ y el contenido de los metabolitos secundarios cuantificados en las especies muestreadas. Para lo anterior, se utilizó el paquete estadístico SPSS, versión 11.5.

\section{Resultados y discusión}

\section{Polifenoles Totales (PFT)}

Estas sustancias constituyen un amplio grupo de compuestos del metabolismo secundario de las plantas, y desempeñan diversas funciones de protección del ataque de patógenos o de organismos herbívoros. También han sido considerados por Hadacek (2002) como productos de desintoxicación, sobrexpresiones del metabolismo primario, productos de degradación y productos de almacenamiento. Son sintetizados a partir de esqueletos de carbono, por lo cual necesitan, y demandan, reservas de carbono para su formación y su crecimiento (Masashi, Takashi y Kouhei, 2007). 
Los polifenoles son los metabolitos secundarios más ampliamente distribuidos dentro del reino vegetal. Se trata de sustancias que poseen al menos un anillo aromático con un radical hidroxilo sustituyente en su estructura química (Makkar y Goodchild, 1996).

Dado que la mayor concentración de polifenoles, tales como los ácidos hidroxibenzóico e hidroxicinámico y los flavonoides, están presentes en las plantas vasculares
(Kumar, citado por García, 2004); estos metabolitos, además de las isoflavonas, tienen una apreciable distribución en las leguminosas, como es el caso de Gliricidia sepium; en relación con especies vegetales diferentes, por ejemplo, la Tithonia diversifolia, posiblemente generará otro tipo de moléculas polifenólicas. Los resultados obtenidos para esta variable, que es analizada en las tres poblaciones objeto de estudio, se muestran en la Figura 1.

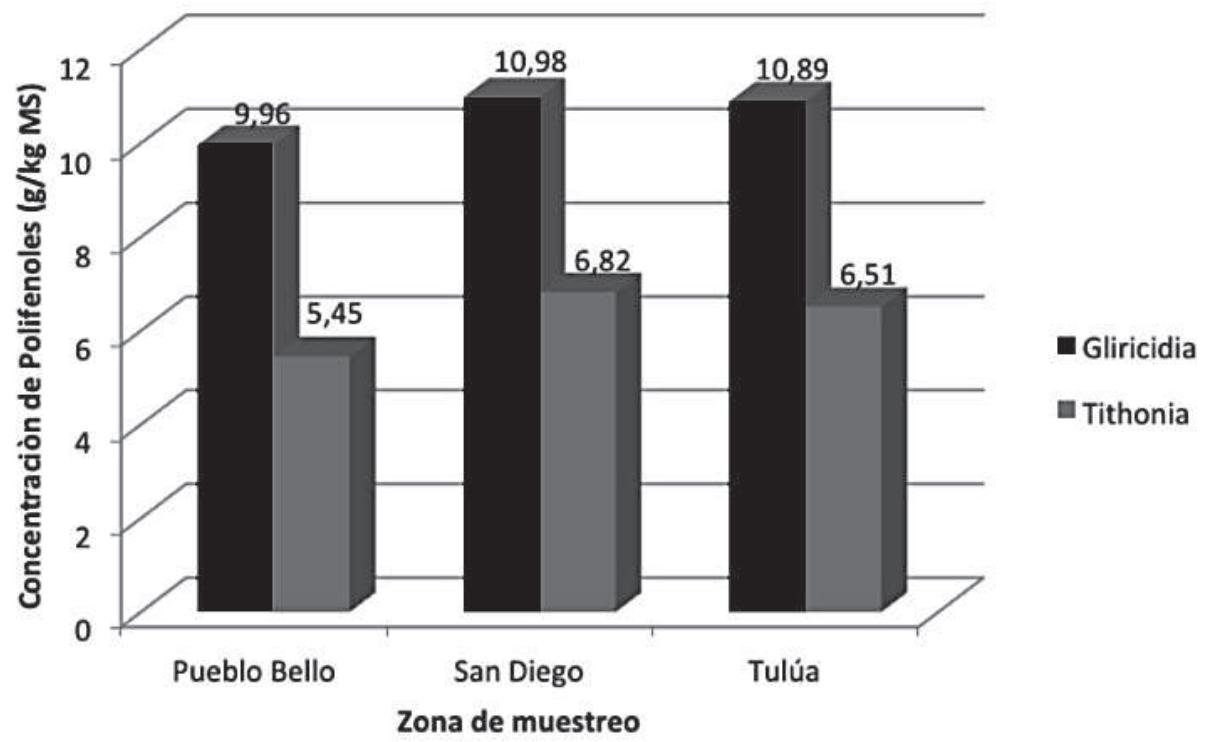

Figura 1. Concentración de polifenoles totales por especie, en las tres zonas muestreadas

En la Figura 1 se aprecia que el contenido de polifenoles en Gliicidia sepium osciló en un rango de 9,96 g. kg-1a 10,98 g. kg-1, y fue similar en las zonas de Tuluá y de Pueblo Bello, y con una diferencia no significativa estadísticamente con la de San Diego; sin embargo, la diferencia, probablemente, se presentó por las condiciones edáficas o de estrés climático propias de la región de San Diego. Al respecto, Salminen, Roslin, Karonen, Sinkkonen, Pihlaja y Pulkkinen (2004), así como Isaza (2007), expresan que los polifenoles suelen incrementarse en situaciones de estrés.

Por su parte, los valores obtenidos en Gliricidia sepium fueron inferiores a los obtenidos por García et al. (2009), los cuales fueron de $22,2 \mathrm{~g} \mathrm{Kg}^{-1}$; estos últimos datos fueron obtenidos en unas condiciones climáticas de $28^{\circ} \mathrm{C}$ y $1650 \mathrm{~mm}$ de precipitación, y el alto contenido de polifenoles puede atribuirse a las altas temperaturas, pues, según García (2009), dentro de los diversos factores que afectan la biosíntesis de estos metabolitos secundarios se destacan el estado acuífero y la temperatura ambiental.

Igualmente, al comparar los datos de la investigación con los reportados por Romero et al. (2000) con la especie
Gliricidia sepium se observa que en este último se obtuvieron valores menores, los cuales fluctuaron entre 2,58 g. $\mathrm{kg}^{-1}-3,56 \mathrm{~g} . \mathrm{kg}^{-1}$ en condiciones de pastoreo y sin pastoreo, respectivamente.

En cuanto a la especie Tithonia diversifolia, no se aprecian diferencias significativas entre las diferentes zonas de muestreo del estudio, las cuales oscilaron entre 5,45 g. $\mathrm{kg}^{-1}-6,82 \mathrm{~g} . \mathrm{kg}^{-1}$, y al comparárselos con los de Verdecia et al. (2011) se hallaron contenidos similares, con fluctuacio-

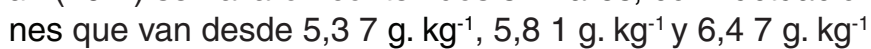
para cortes de 60, 120 y 180 días, respectivamente.

Si bien con la especie Tithonia diversifolia no se observaron diferencias estadísticas significativas en el contenido de polifenoles, ya que en San Diego fue mayor; probablemente, por los altos niveles de temperatura en esta región, lo cual coincide con las conclusiones de Salminen et al. (2004), quienes expresaron que el contenido fenólico foliar aumenta durante el verano, independientemente de la textura del suelo. Por lo anterior, se atribuyen a dichos compuestos funciones de defensa contra los depredadores, los patógenos y la irradiación solar. 


\section{Taninos totales}

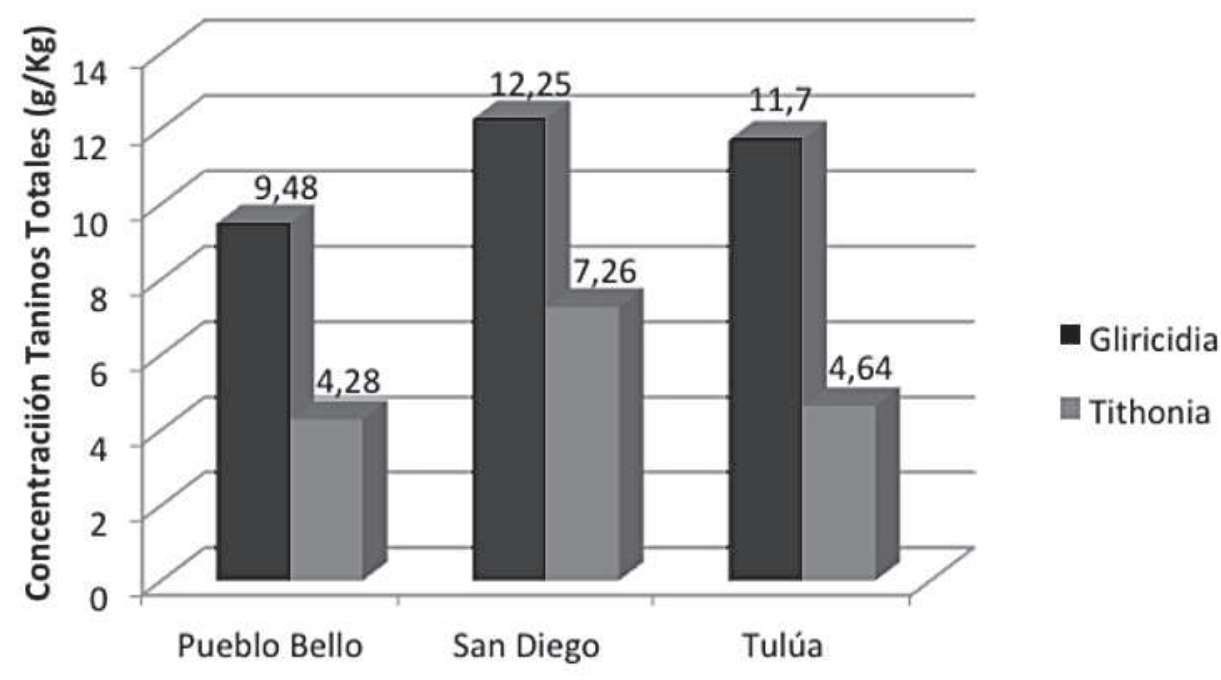

Zonas muestreadas

Figura 2. Concentración de taninos totales por especie en las tres zonas muestreadas

Los taninos se definen como compuestos polifenólicos solubles en agua, de estructura química variada y ampliamente distribuidos en el mundo vegetal. Son de alto peso molecular (500-3000 Daltons) y se localizan en las vacuolas, combinados con alcaloides y proteínas; desempeñan así una función defensiva frente a insectos y hongos (Makkar, 1997; Kumar y D'Mello, 1995; Peris, 1995; Makkar y Goodchild, 1996). Las plantas varían cualitativamente en su síntesis como respuesta a los cambios ambientales y fisiológicos (Giner Chávez, 1996).
En el presente estudio se evidenciaron diferencias en el contenido de taninos totales en la especie Gliricidia sepium en las zonas muestreadas, y ello ratifica el comportamiento de un mayor contenido de metabolitos en zonas de altas temperaturas. En la especie Tithonia diversifolia se apreció la misma tendencia.

\section{Taninos condensados}

Los taninos condensados son polímeros de unidades de flavonoides unidas por enlaces $\mathrm{C}-\mathrm{C}$, los cuales no pueden ser hidrolizados, pero sí oxidados, por un ácido fuerte, para rendir antocianidinas.

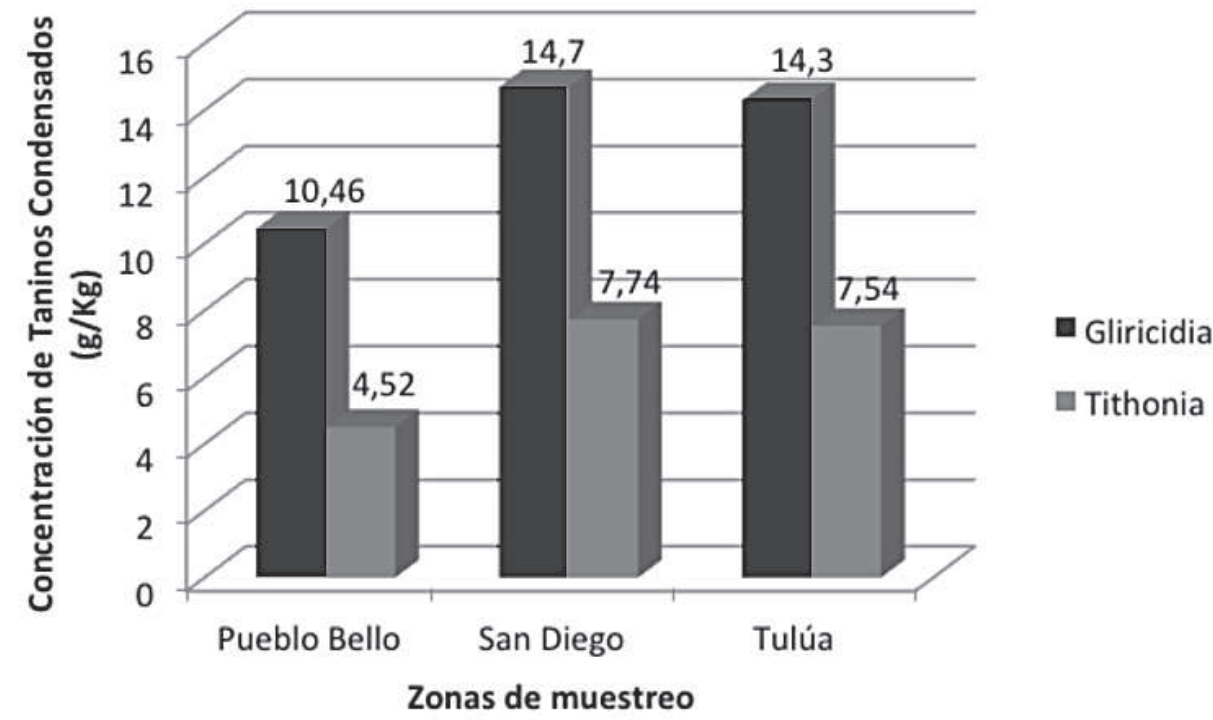

Figura 3. Concentración de taninos condensados por especie en las tres zonas muestreadas 
Respecto al contenido de taninos condensados en la especie Gliricidia sepium en las zonas muestreadas, se presentó un mayor contenido de este metabolito en zonas de altas temperaturas. En la especie Tithonia diversifolia se aprecia el mismo comportamiento (Figura 3).

\section{Saponinas}

Las saponinas son glucósidos que se encuentran distribuidos ampliamente en las plantas, y están formadas por una aglicona de origen terpénico, esteroidal o esteroidal alcaloide, al cual se une por el hidroxilo del carbono-3 una cadena ramificada de azúcares, que, a su vez, puede ser de hasta cinco moléculas; usualmente, glucosa, arabinosa, ácido glucurónico, xilosa y ramnosa (Díaz, 2009). Las saponinas esteroidales se hallan, principalmente, en las monocotiledóneas, mientras que las saponinas terpénicas se encuentran, sobre todo, en las dicotiledóneas (Muller, 2009).

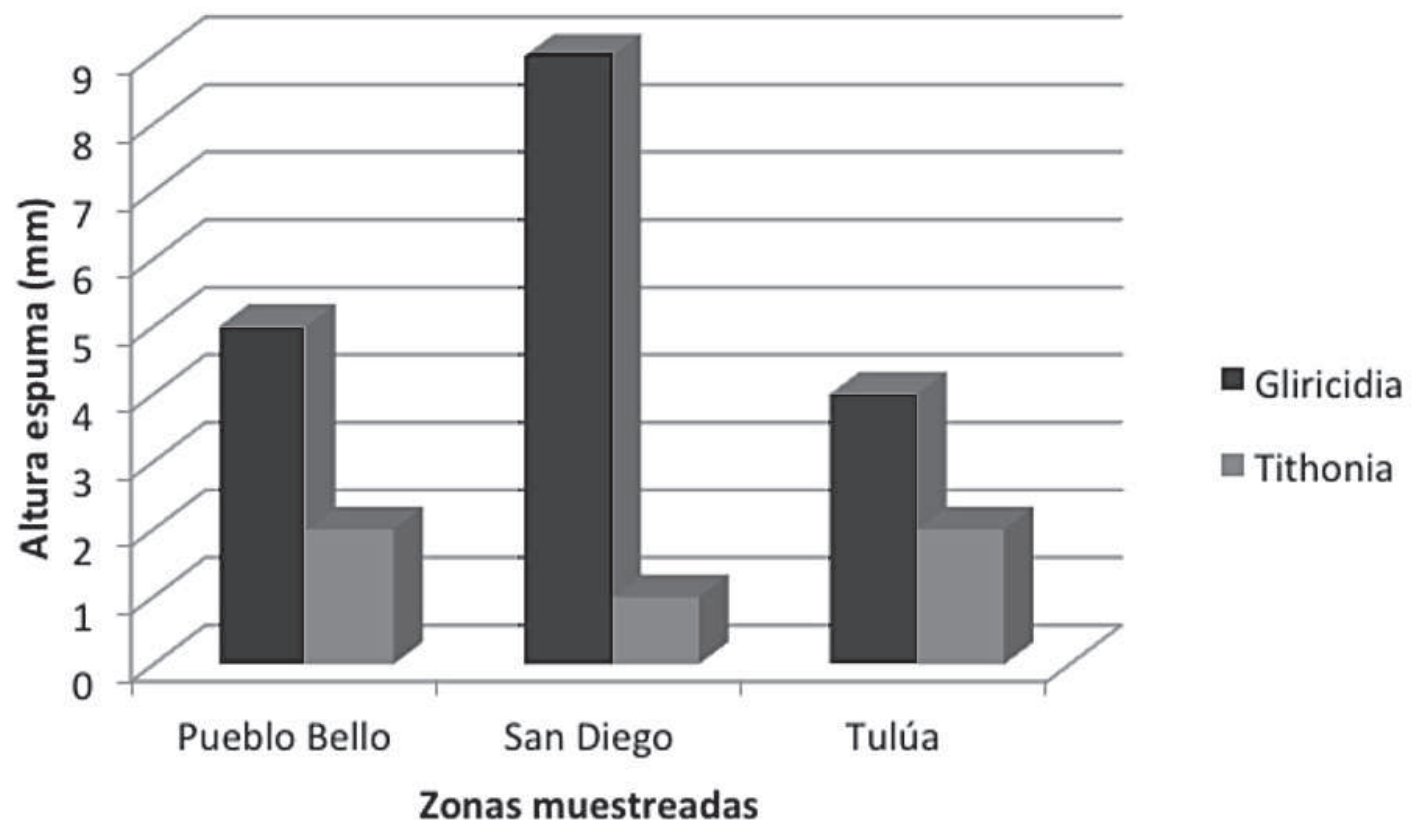

Figura 4. Concentración de saponinas por especie en las tres zonas muestreadas

Respecto al contenido de saponinas en la especie Gliricidia sepium en las zonas muestreadas, se presentó, igualmente, un mayor contenido en zonas de altas temperaturas. En la especie Tithonia diversifolia no se aprecia la misma tendencia (Figura 4).

\section{Correlación entre la conductividad eléctrica y la con- centración de taninos y saponinas}

Teniendo presente que la conductividad eléctrica (CE) del suelo es un valor correlacionado con algunas propiedades del suelo que afectan la productividad de las cosechas, tales como la textura, la capacidad de intercambio catiónico, las condiciones de drenaje, el contenido de materia orgánica, la salinidad y las características del subsuelo, el estudio muestra que existe una alta correlación entre la CE y el contenido de polifenoles totales en la especie Gliricidia sepium. Por su parte, en la Tithonia diversifolia se observa este mismo comportamiento. Lo anterior coincide con lo expuesto por Arámbula et al. (2010), quienes hallaron una correlación alta y positiva entre el contenido fenólico y la conductividad eléctrica en suelos de textura arcilloarenosa.

La Figura 5 presenta un consolidado de la relación cuantitativa entre conductividad eléctrica, $\mathrm{pH}$ y contenido de fenoles y taninos, en las especies analizadas. 


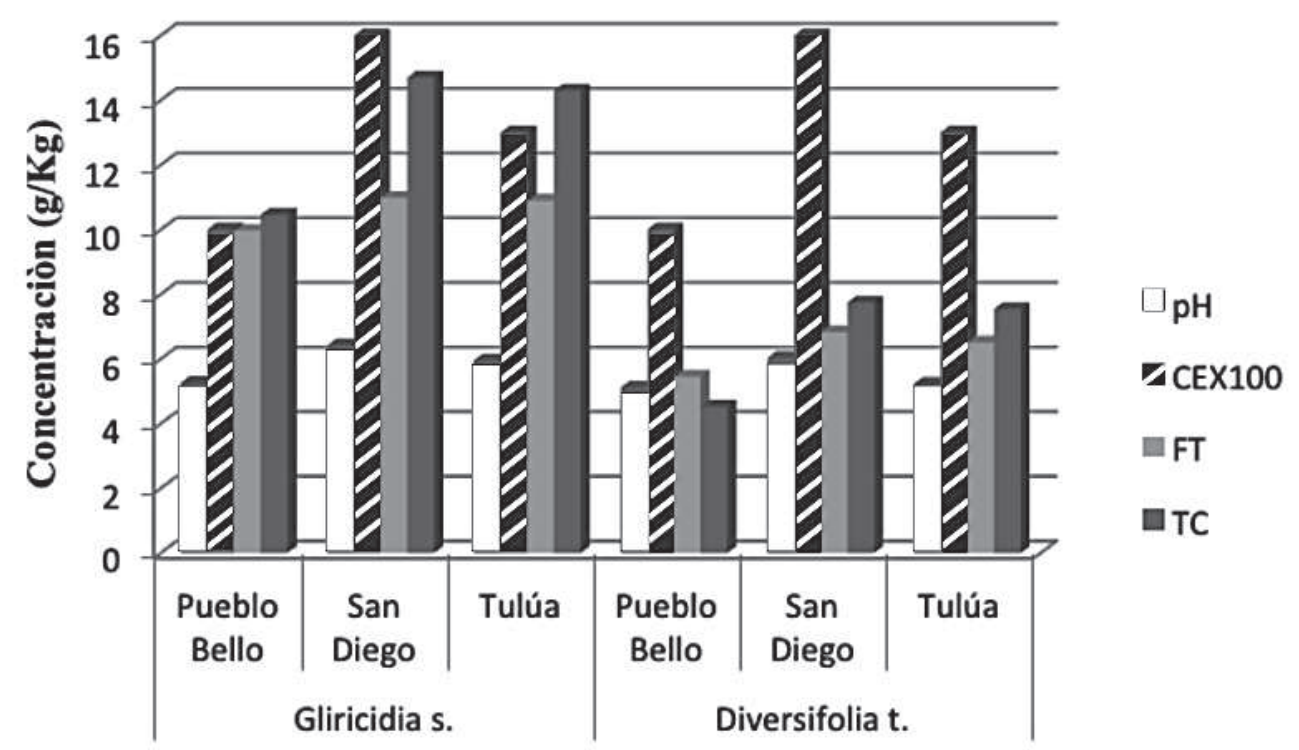

Zonas y especies muestreadas

Figura 5. Relación entre conductividad eléctrica (CE) y pH del suelo y el contenido de fenoles totales, taninos condensados, taninos totales y saponinas en las especies estudiadas

En la anterior figura se puede evidenciar la alta correlación positiva entre la conductividad eléctrica y los diferentes tipos de metabolitos (fenoles totales, taninos condensados y taninos totales) en las especies estudiadas, lo cual se puede atribuir a la mayor actividad del metabolismo vegetal en condiciones de alta conductividad eléctrica (Figura 5).

Cuanto más elevada sea la concentración de sales solubles en el suelo se tendrá una mayor conductividad eléctrica, o capacidad del agua para transportar la corriente eléctrica; por ende, este indicador es una forma indirecta de medir la salinidad del suelo, así como el contenido de sólidos disueltos. Esto último explicaría la alta correlación entre la conductividad eléctrica y la producción de metabolitos secundarios en tejidos vegetales.

Respecto a los taninos condensados, se observa que, igualmente, hay una correlación positiva entre la CE y sus niveles en las especies vegetales estudiadas, como se observa en la Tabla 4.

Tabla 4. Análisis de la correlación entre la conductividad eléctrica y los niveles de metabolitos en las especies vegetales estudiadas

\begin{tabular}{|c|c|c|c|c|c|c|}
\hline & $\mathrm{pH}$ & CE & FT & TC & TT & SAP(mm) \\
\hline $\mathrm{pH}$ & 1 & & & & & \\
\hline CE & 0,86323157 & 1 & & & & \\
\hline FT & 0,59542893 & 0,21798096 & 1 & & & \\
\hline $\mathrm{TC}$ & 0,72642553 & 0,41218632 & 0,96680532 & 1 & & \\
\hline TT & 0,75000894 & 0,373703 & 0,97032675 & 0,96364729 & 1 & \\
\hline $\begin{array}{l}\text { SAP } \\
(\mathrm{mm})\end{array}$ & 0,65673935 & 0,38198743 & 0,84408718 & 0,83945188 & 0,84127243 & 1 \\
\hline
\end{tabular}




\section{Conclusiones}

Existe una alta correlación positiva entre la conductividad eléctrica y los diferentes tipos de metabolitos (fenoles totales, taninos condensados y taninos totales) en las especies Gliricidia sepium y Thitonia diversifolia, lo cual se puede atribuir a la mayor actividad del metabolismo vegetal en condiciones de alta conductividad eléctrica.

Se ha encontrado que mientras más elevada sea la concentración de sales solubles en el suelo se tiene una mayor conductividad eléctrica, o capacidad del agua para transportar la corriente eléctrica, por lo cual este indicador es una forma indirecta de medir la salinidad del suelo, al igual que el contenido de sólidos disueltos.

La conductividad eléctrica (CE) del suelo es un valor correlacionado con algunas propiedades del suelo que afectan la productividad de las cosechas, incluyendo la textura, la capacidad de intercambio catiónico, las condiciones de drenaje, el contenido de materia orgánica, la salinidad y las características del subsuelo.

\section{Literatura citada}

1. AOAC. (1995). Official Method of Analysis of the Associations of Official Analytical Chemists. Vol. II W. Horwitz (Eds.). Washington, D. C.

2. $A O A C$ (2005). Association of Official Analytical Chemists. Official methods of analysis of the AOAC. 18th. Ed. AOAC, Arlington, VA.

3. Arámbula, J. Ibarra, B. I., González, B., Galindo, O.D., Hernández, H. 2010. Variación estacional de compuestos fenólicos foliares en Quersus sideroxila en diferentes tipos de suelo. Madera y Bosques 16(3), 49-59. Instituto de ecología, A.C, Xalapa (México)

4. Ashok, K. .J., Vincent R.M y Nessler 2000. Molecular Characterization of a hydroxyl Methylglutaryl Coareductase gene from Mulberry (Morus Alba L). Plant mol Biol 42:559

5. Baxter, N. J. 1997. Multiple Interactions between Polyphenols and salivary proline rich protein result in complexation and precipitation. Journal of Biochemistry. Yorkshire U.K. 36 (19): 503

6. Cano, R., Carulla, J. \& Lascano, C. 1994 Métodos de conservación de muestras de forraje de leguminosas tropicales y su efecto en el nivel y en la actividad biológica de los taninos. Pasturas tropicales. 16 (1):2-7.

7. Cuellar, A., Márquez, I., Hernández, J., Alemán, A. 1999. Estudio fotoquímico de la especie hibiscus elatus s.w. Rev Cubana Farm. 33(2):127-31.

8. Dewanto, V., Wu, K., Adom, R. ( 2002). Thermal processing enhances the nutritional value of tomatoes by increasing total antioxidant activity. J. Agric. Food Chem. 59: 3010-3014.

9. Diaz, L. 2009. Interacciones moleculares entre plantas y microrganismos: Saponinas como defensas químicas de las plantas y su tolerancia a los microrganismos; Una revisión: RET: Revista de Estudios Transdisciplinarios, vol. 1 (2): 32-55.

10. Flores, O., Ibrahlm, M., Kass, D., \& Andrade, H. 1999. El efecto de los taninos en especies leñosas forrajeras sobre la utilización de nitrógeno por bovinos Revista Agroforestería en las Américas. 6(23). http://web.catie.ac.cr/ informacion/RAFA/

11. Gershenzon, J. 1984. Changes in the levels of plant secondary metabolites under water water and nutrient stress. Recent Advances in Phytochemistry 18:273-320

12. García, D.E. 2004. Los metabolitos secundarios de las especies vegetales. Pastos y Forrajes, Vol. 27, No. 1.

13. García, M., García, G., Sanabria, M. 2010 Efecto de la salinidad sobre el crecimiento, daño oxidativo y concentración foliar de metabolitos secundarios en dos variedades de Caraota (Phaseolus vulgaris). Revista Interciencia Caracas (Venezuela). Vol 35, (11): 840-846.

14. Giner, B. 1996. Condensed tannins in tropical foragesPH. D. . Thesis. Cornel University, Ithaca, NY. USA.

15. Gutiérrez, V. E., Villaseñor, A. A., Cancino, M.R., Lemus O. E. \& Madrigal. S. 2003. Contenido de compuestos fenólicos en arbustos y árboles forrajeros en San Lucas, Michoacán. XIV Encuentro de Investigación Pecuaria y Producción Animal Morelia, Michoacán p182-186.

16. Haukioja, E., Niemelä, P. \& Sirén, S. 1995. Foliage phenols and nitrogen in relation to growth, insect damage, and ability to recover after defoliation, in the mountain birch Betula pubescens, spp tortuosa. o ecologia. 65:214-222.

17. Hadacek, F. 2002. Secondary metabolites as plant traits: current assessment and future perspectives. Critical Reviews in Plant Science (21):273-322.

18. Harborne, J. B.1993 :- Introduction to Ecological Biochemestry. 4th Edición.Academic Press, Harcomt Brace \& Co. Publishers, New York, USA. 320 p.

19. Isaza,M. J..H. 2007. Taninos o Polifenoles Vegetales. Universidad Tecnológica de Pereira, Colombia. Scientia et Technica. Año XIII (33):13-18.

20. Instituto Geográfico Agustín Codazzi-IGAG, 2006. Métodos analíticos de laboratorio de suelos. Bogotá, Colombia.

21. Jackson, F. S., Barry, C., Lascano, C. \& Palmer, B. 1996. The Extractable, and Bound Condensed Tannin Content of Leaves for Tropical Tree, Shrub and Forage Legumes. J. of Science of Food and Agriculture. New Zealand. 17 (1): 103-110.

22. Kaito, R. J. Umunna, N. N., Nsahlai, I. V., Tamminga, S., Vabruchem, J.,Hanson, J.\& Vandewouw, M. 1996. Palatability of multipurpose tree species. Effect of species 
and length of study on intake and relative palatability by sheep. Agroforestry Systems 33(3):249-261

23. Kumar, R. 1997. Ant nutritional factors. The potential risks of toxicity and the methods to alleviate them. In: Legumes trees and other fodder trees as protein source for livestock. (Eds. Speedy).

24. Leinmúller, E., H, Steingass, H \& K.H. Menke. 1995. Tannins in ruminant feedstuffs. Animal and Research and Develovment 33: 9-62.

25. Makkar, H. P., Blümmel, M. \& Becker, k. 1997. Formation of complexes between polyvinyl pyrrolidones or polyethylene glycols and tannins, and their implication in gas production and true digestibility in vitro techniques. British Journal of Nutrition. 73: 897-913.

26. Makkar, H. P.\&Goodchild, V. A. 1997. Quantification of tannins a laboratory manual. pasture, forage and livestock program International Center for Agricultural research in the dry areas. Second edition.Alepo, Syria.

27. Masashi, K. N., Takashi, H., Kouhei, L. 2007. Amongtree variation in leaf and herbivore attacks in a deciduous oak. Quercusdentate. Scandinavian Journal or Forestry Research 22:221- 218.

28. Mungarulire, J., Franz, C.H.,, Seitz, R. \& Verlet, N. 1993. Some developments in the search for cytotoxic constituents from Rwandese medicinal plants. Acta horticulturae 333: 211-216.

29. Muller, C. 2009. Role of glucosinolates in plant invasiveness. Phytochemistry Reviews, 8 (1), 227-242.

30. Palma, J. M., Aguirre, M., Cárdenas, M. \& Moya, A. 1999. Valor nutritivo de tres leguminosas arbóreas en el trópico seco de México. Pastos y Forrajes 22:57-63

31. Oncina, R., J.M., Botía, J.A., Del Río \& Ortuño, A. 2000. Bioproduction of diosgenin in callus cultures of Trigonella foenum-graecum L. Food Chemistry 70(4): 489492.

32. Posada, S., Montoya, G., Ceballos, A. (2005). Efecto de los taninos sobre la digestión, el metabolismo y la producción en rumiantes" Bioquímica, Nutrición y Alimentación De La Vaca .En:Colombia ISBN: 9583372773 ed: Biogénesis , v. , p. $181-206$.
33. Romero, L. C. E., Palma, G. J. M. \& López, J. 2000. Influencia del pastoreo en la concentración de fenoles y taninos condensados en Gliricidia sepium en el trópico seco. Livestock Research for Rural Development 4(12):1-9 http://www.cipav.org.co/lrrd//rrd12/4/rome124.htm

34. Rosales M.M. 1998. Mezcla de forrajes, uso de la biodiversidad forrajera tropical en sistemas agroforestales; Memorias de una Conferencia Electrónica; Agroforestería para la Producción Animal en Latinoamérica; FAO-CIPAV; Cali, Colombia.

35. Salminen, J. P., Roslin, M., Karonen, J., Sinkkonen, K., Pihlaja y P. Pulkkinen. 2004. Seasonal variation in the content of hydrolyzable tannins, flavonoid glycosides, and proanthocyanidins in oak leaves. J Chem Ecology. 30(9):1693- 1711.

36. Terril, T. H., Rowan, A.M., Douglas. G.B., \& Barry,T.N.1992..Determination of extractable and bound condensed tannin concentration in forage plants, protein concentrate meals and cereal grains. J.Sci. Food agric.58:321-329

37. Vargas J E. 1994. Caracterización de recursos forrajeros disponibles en tres agroecosistemas del Valle del Cauca. En: Memorias II Seminario Internacional Desarrollo sostenible de Sistemas Agrarios, Maestría en Sistemas Sostenibles de Producción Animal en los Trópicos. Cali. p 135-149.

38. Verdecia, D. M., Ramírez, J.L.,Leonard, I., Álvarez, Y., Bazán, Y., Bodas, R. Andrés, S., Álvarez, J., Giráldez, F., López, S. 2011, REDVET. Revista electrónica de veterinaria. Calidad de la Tithonia diversifolia en una zona del Valle del Cauto. 12(5): 13, 31.

39. Waterman, P.G., Mole, S.. (1994): Method in Ecology. Analysis of Phenolic Plant Metabolites. Blackwell Sci. Publ., London: 66-103.

40. Wanjau, S., Mukalama, J. \& Thijssen, R. 1998. Transferencia de biomasa: Cosecha gratis de fertilizante. Boletín de ILEIA. Marzo de 1998. p 25.

Recibido: 1 de marzo de 2012. Aceptado: 20 de abril de 2012. 